\title{
The Basement Flight
}

The approach to clinical conundrums by an expert clinician is revealed through the presentation of an actual patient's case in an approach typical of a morning report. Similar to patient care, sequential pieces of information are provided to the clinician, who is unfamiliar with the case. The focus is on the thought processes of both the clinical team caring for the patient and the discussant.

This icon represents the patient's case. Each paragraph that follows represents the discussant's thoughts.

Chetna K Pande, MD, MPH${ }^{1}$; Justin Berk, MD, MPH, MBA ${ }^{1,2}$; Mandeep S Jassal, MD, MPH ${ }^{1}$; Reza Manesh, MD*; Andrew PJ Olson, MD³

'Department of Pediatrics, The Johns Hopkins Hospital, Baltimore, Maryland; '2Department of Medicine, The Johns Hopkins Hospital, Baltimore, Maryland; ${ }^{3}$ Departments of Medicine and Pediatrics, University of Minnesota Medical School, Minneapolis, Minnesota.

A 14-year-old girl with a history of asthma presented to the Emergency Department (ED) with three months of persistent, nonproductive cough, and progressive shortness of breath. She reported fatigue, chest tightness, orthopnea, and dyspnea with exertion. She denied fever, rhinorrhea, congestion, hemoptysis, or paroxysmal nocturnal dyspnea.

Her age and past medical history of asthma are incongruent with her new symptoms, as asthma is typified by intermittent exacerbations, not progressive symptoms. Thus, another process, in addition to asthma, is most likely present; it is also important to question the accuracy of previous diagnoses in light of new information. Her symptoms may signify an underlying cardiopulmonary process, such as infiltrative diseases (eg, lymphoma or sarcoidosis), atypical infections, genetic conditions (eg, variant cystic fibrosis), autoimmune conditions, or cardiomyopathy. A detailed symptom history, family history, and careful physical examination will help expand and then refine the differential diagnosis. At this stage, typical infections are less likely.

She had presented two months prior with nonproductive cough and dyspnea. At that presentation, her temperature was $36.3^{\circ} \mathrm{C}$, heart rate 110 beats per minute, blood pressure $119 / 63 \mathrm{~mm} \mathrm{Hg}$, respiratory rate 43 breaths per minute, and oxygen saturation $86 \%$ while breathing ambient air. A chest CT with contrast demonstrated diffuse patchy multifocal ground-glass opacities in the bilateral lungs as well as a mixture of atelectasis and lobular emphysema in the dependent lobes bilaterally (Figure 1). Her main pulmonary artery was dilated at $3.6 \mathrm{~cm}$ (mean of $2.42 \mathrm{~cm}$ with SD 0.22). She

*Corresponding Author: Reza Manesh, MD; E-mail: rsedigh1@jhmi.edu Telephone: 412-708-6944;

Published online first November 28, 2018

Received: April 13, 2018; Revised: August 13, 2018;

Accepted: September 7, 2018

(C) 2019 Society of Hospital Medicine DOI 10.12788/jhm.3096 was diagnosed with atypical pneumonia. She was administered azithromycin, weaned off oxygen, and discharged after a seven-day hospitalization.

Two months prior, she had marked tachypnea, tachycardia, and hypoxemia, and imaging revealed diffuse ground-glass opacities. The differential diagnosis for this constellation of symptoms is extensive and includes many conditions that have an inflammatory component, such as atypical pneumonia caused by Mycoplasma or Chlamydia pneumoniae or a common respiratory virus such as rhinovirus or human metapneumovirus. However, two findings make an acute pneumonia unlikely to be the sole cause of her symptoms: underlying emphysema and an enlarged pulmonary artery. Emphysema is an uncommon finding in children and can be related to congenital or acquired causes; congenital lobar emphysema most often presents earlier in life and is focal, not diffuse. Alpha-1-anti-trypin deficiency and mutations in connective tissue genes such as

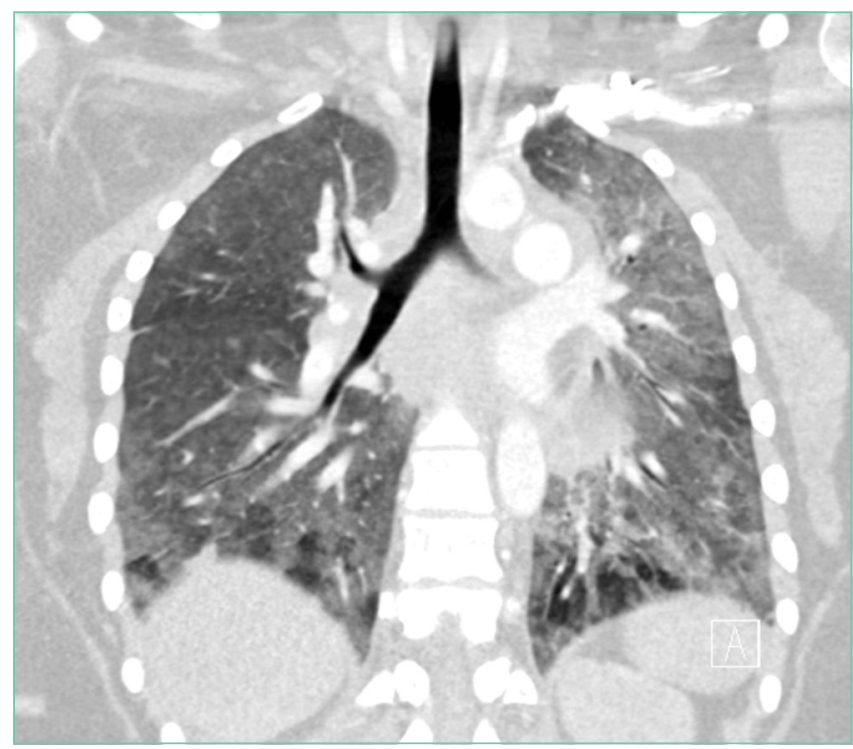

FIG 1. Coronal Chest CT demonstrating diffuse patchy ground-glass opacities in the left lung, particularly the left upper lobe. Multifocal ground-glass opacities are also noted throughout the right lung. 


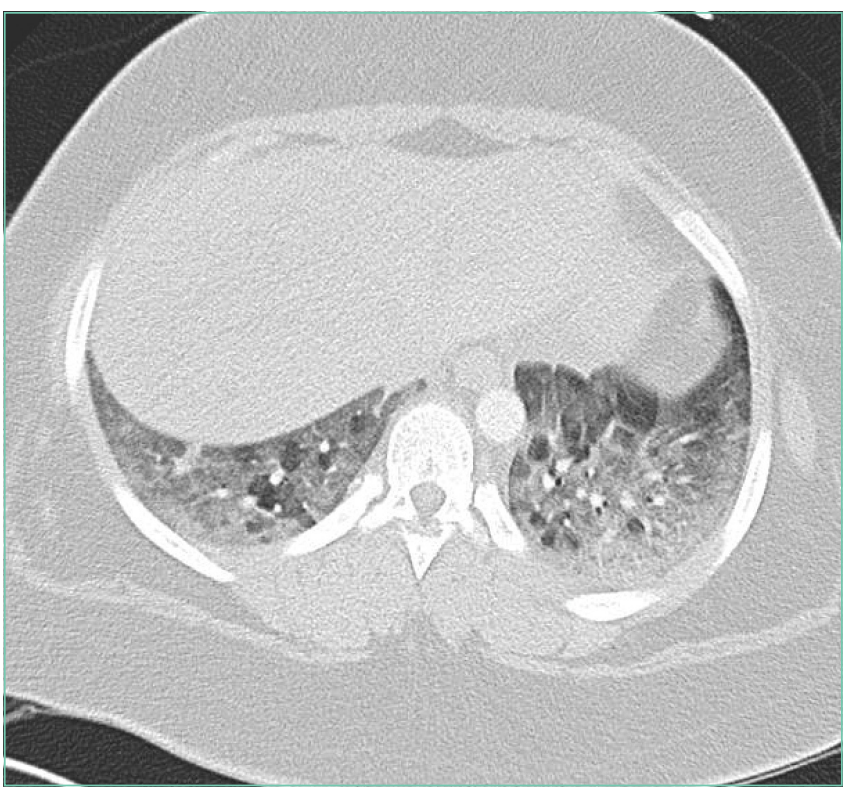

FIG 2. Axial Chest CT demonstrating diffuse centrilobular ground-glass opacities and patchy areas of lobular air-trapping with mosaic attenuation seen most prominently in the bilateral lower lobes.

those encoding for elastin and fibrillin can lead to pulmonary disease. While not diagnostic of pulmonary hypertension, her dilated pulmonary artery, coupled with her history, makes pulmonary hypertension a strong possibility. While her pulmonary hypertension is most likely secondary to chronic lung disease based on the emphysematous changes on the CT, it could still be related to a cardiac etiology.

The patient had a history of seasonal allergies and well-controlled asthma. She was hospitalized at age six for an asthma exacerbation associated with a respiratory infection. She was discharged with an albuterol inhaler, but seldom used it. Her parents denied any regular coughing during the day or night. She was morbidly obese. Her tonsils and adenoids were removed to treat obstructive sleep apnea (OSA) at age seven, and a subsequent polysomnography was normal. Her medications included intranasal fluticasone propionate and oral iron supplementation. She had no known allergies or recent travels. She had never smoked. She had two pet cats and a dog. Her mother had a history of obesity, OSA, and eczema. Her father had diabetes and eczema.

The patient's history prior to the recent few months sheds little light on the cause of her current symptoms. While it is possible that her current symptoms are related to the worsening of a process that had been present for many years which mimicked asthma, this seems implausible given the long period of time between her last asthma exacerbation and her present symptoms. Similarly, while tonsillar and adenoidal hypertrophy can be associated with infiltrative diseases (such as lymphoma), this is less common than the usual (and normal) disproportionate increase in size of the adenoids compared to other airway structures during growth in children.
She was admitted to the hospital. On initial examination, her temperature was $37.4^{\circ} \mathrm{C}$, heart rate 125 beats per minute, blood pressure $143 / 69 \mathrm{~mm} \mathrm{Hg}$, respiratory rate 48 breaths per minute, and oxygen saturation $86 \%$ breathing ambient air. Her BMI was $58 \mathrm{~kg} / \mathrm{m} 2$. Her exam demonstrated increased work of breathing with accessory muscle use, and decreased breath sounds at the bases. There were no wheezes or crackles. Cardiovascular, abdominal, and skin exams were normal except for tachycardia. At rest, later in the hospitalization, her oxygen saturation was $97 \%$ breathing ambient air and heart rate $110 \mathrm{bpm}$. After two minutes of walking, her oxygen saturation was $77 \%$ and heart rate $132 \mathrm{bpm}$. Two minutes after resting, her oxygen saturation increased to $91 \%$.

Her white blood cell count was $11.9 \times 10^{9} / \mathrm{L}$ (67\% neutrophils, $24.2 \%$ lymphocytes, $6 \%$ monocytes, and $2 \%$ eosinophils), hemoglobin $11.2 \mathrm{~g} / \mathrm{dL}$, and platelet count 278,000/ $\mathrm{mm}^{3}$. Her complete metabolic panel was normal. The C-reactive protein (CRP) was $24 \mathrm{mg} / \mathrm{L}$ (normal range, < 4.9) and erythrocyte sedimentation rate (ESR) $103 \mathrm{~mm} /$ hour (normal range, 0-32). A venous blood gas (VBG) showed a $\mathrm{pH}$ of 7.42 and pCO2 39. An EKG demonstrated sinus tachycardia.

The combination of the patient's tachypnea, hypoxemia, respiratory distress, and obesity is striking. Her lack of adventitious lung sounds is surprising given her CT findings, but the sensitivity of chest auscultation may be limited in obese patients. Her laboratory findings help narrow the diagnostic frame: she has mild anemia and leukocytosis along with significant inflammation. The normal $\mathrm{CO}_{2}$ concentration on VBG is concerning given the degree of her tachypnea and reflects significant alveolar hypoventilation.

This marked inflammation with diffuse lung findings again raises the possibility of an inflammatory or, less likely, infectious disorder. Sjogren's syndrome, systemic lupus erythematosus (SLE), and juvenile dermatomyositis can present in young women with interstitial lung disease. She does have exposure to pets and hypersensitivity pneumonitis can worsen rapidly with continued exposure. Another possibility is that she has an underlying immunodeficiency such as common variable immunodeficiency, although a history of recurrent infections such as pneumonia, bacteremia, or sinusitis is lacking.

An echocardiogram should be performed. In addition, laboratory evaluation for the aforementioned autoimmune causes of interstitial lung disease, immunoglobulin levels, pulmonary function testing (if available as an inpatient), and potentially a bronchoscopy with bronchoalveolar lavage (BAL), and biopsy should be pursued. The BAL and biopsy would be helpful in evaluating for infection and interstitial lung disease in an expeditious manner.

A chest CT without contrast was done and compared to the scan from two months prior. New diffuse, ill-defined centrilobular ground-glass opacities were evident throughout the lung fields; dilation of the main pulmonary artery was unchanged, and previously seen ground-glass opacities had 

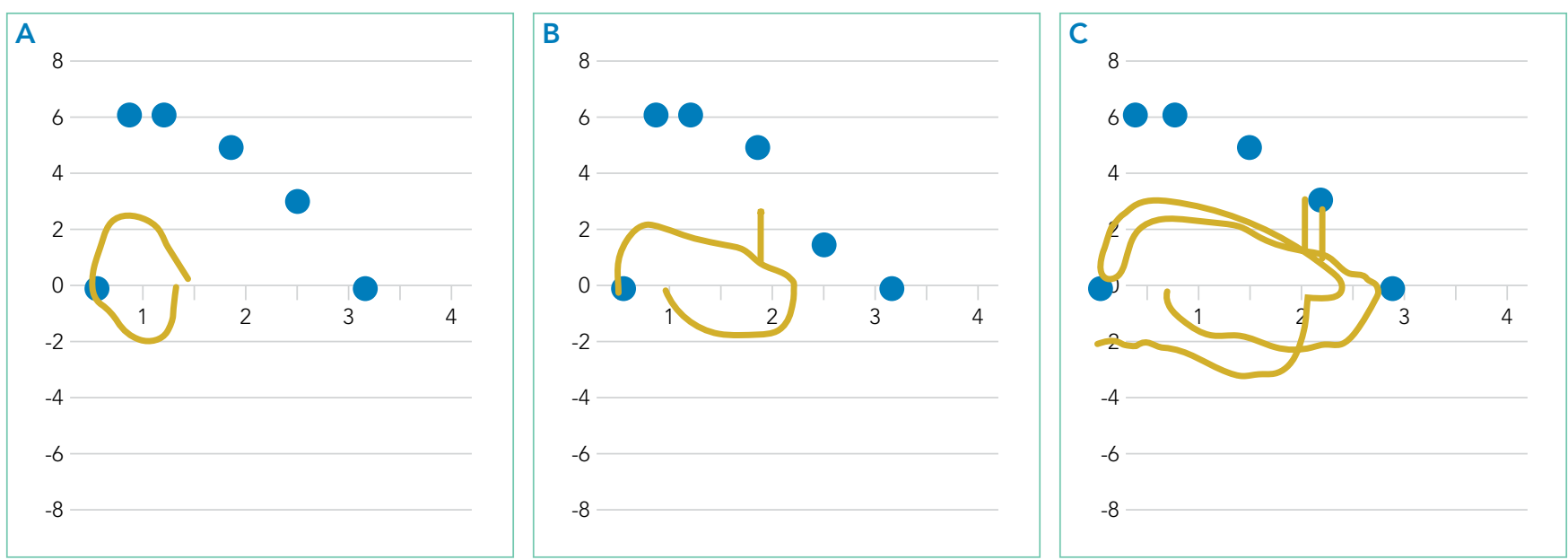

FIG 3. Spirometric Flow Volume Loops Before and After Exposure Removal. (A) At admission: FVC 1.07L (35\% predicted); FEV1: 1.07L (39\% predicted); FEV1/FVC 100\%. (B) two months postremoval: FVC 2.00L (66\% predicted); FEV1: 188L (69\% predicted); FEV1/FVC: $94 \%$. (C) 6 months postremoval: FVC: 2.74 L (95\% predicted); FEV1: 2.03L (78\% predicted), FEV1/FVC: 74\%.

resolved. There were patchy areas of air-trapping and mosaic attenuation in the lower lobes (Figure 2). Transthoracic echocardiogram demonstrated a right ventricular systolic pressure of $58 \mathrm{~mm} \mathrm{Hg}$ with flattened intraventricular septum during systole. Left and right ventricular systolic function were normal. The left ventricular diastolic function was normal. Pulmonary function testing demonstrated a FEV1/FVC ratio of $100(112 \%$ predicted), FVC $1.07 \mathrm{~L}$ (35\% predicted) and FEV1 $1.07 \mathrm{~L}$ (39\% predicted), and total lung capacity was $2.7 \mathrm{~L}$ (56\% predicted) (Figure 3). Single-breath carbon monoxide uptake in the lung was not interpretable based on 2017 European Respiratory Society (ERS)/American Thoracic Society (ATS) technical standards.

This information is helpful in classifying whether this patient's primary condition is cardiac or pulmonary in nature. Her normal left ventricular systolic and diastolic function make a cardiac etiology for her pulmonary hypertension less likely. Further, the combination of pulmonary hypertension, a restrictive pattern on pulmonary function testing, and findings consistent with interstitial lung disease on cross-sectional imaging all suggest a primary pulmonary etiology rather than a cardiac, infectious, or thromboembolic condition. While chronic thromboembolic hypertension can result in nonspecific mosaic attenuation, it typically would not cause centrilobular ground-glass opacities nor restrictive lung disease. Thus, it seems most likely that this patient has a progressive pulmonary process resulting in hypoxia, pulmonary hypertension, centrilobular opacities, and lower-lobe mosaic attenuation. Considerations for this process can be broadly categorized as one of the childhood interstitial lung disease (chILD). While this differential diagnosis is broad, strong consideration should be given to hypersensitivity pneumonitis, chronic aspiration, sarcoidosis, and Sjogren's syndrome. An intriguing possibility is that the patient's "response to azithromycin" two months prior was due to the avoidance of an inhaled antigen while she was in the hospital; a detailed environmental history should be explored. The normal poly- somnography after tonsilloadenoidectomy makes it unlikely that OSA is a major contributor to her current presentation. However, since the surgery was seven years ago, and her BMI is presently $58 \mathrm{~kg} / \mathrm{m}^{2}$ she remains at risk for OSA and obesity-hypoventilation syndrome. Polysomnography should be done after her acute symptoms improve.

She was started on $5 \mathrm{~mm} \mathrm{Hg}$ of continuous positive airway pressure (CPAP) at night after a sleep study on room air demonstrated severe OSA with a respiratory disturbance index of 13 events per hour. Antinuclear antibodies (ANA), anti-neutrophil cytoplasmic antibody (ANCA), anti-Jo-1 antibody, anti-RNP antibody, anti-Smith antibody, anti-Ro/SSA and anti-La/SSB antibody were negative as was the histoplasmin antibody. Serum angiotensin-converting enzyme (ACE) level was normal. Mycoplasma IgM and IgG were negative. $\operatorname{lgE}$ was $529 \mathrm{kU} / \mathrm{L}$ (normal range, <114).

This evaluation reduces the likelihood the patient has Sjogren's syndrome, SLE, dermatomyositis, or ANCA-associated pulmonary disease. While many patients with dermatomyositis may have negative serologic evaluations, other findings usually present such as rash and myositis are lacking. The negative ANCA evaluation makes granulomatosis with polyangiitis and microscopic polyangiitis very unlikely given the high sensitivity of the ANCA assay for these conditions. ANCA assays are less sensitive for eosinophilic granulomatosis with polyangiitis (EGPA), but the lack of eosinophilia significantly decreases the likelihood of EGPA. ACE levels have relatively poor operating characteristics in the evaluation of sarcoidosis; however, sarcoidosis seems unlikely in this case, especially as patients with sarcoidosis tend to have low or normal IgE levels. Patients with asthma can have elevated IgE levels. However, very elevated IgE levels are more common in other conditions, including allergic bronchopulmonary aspergillosis (ABPA) and the Hyper-lgE syndrome. The latter manifests with recurrent infections and eczema, and is inherited in an autosomal dominant manner. 
However, both the Hyper-lgE syndrome and ABPA have much higher IgE levels than seen in this case. Allergen-specific IgE testing (including for antibodies to Aspergillus) should be sent. It seems that an interstitial lung disease is present; the waxing and waning pattern and clinical presentation, along with the lack of other systemic findings, make hypersensitivity pneumonitis most likely.

The family lived in an apartment building. Her symptoms started when the family's neighbor recently moved his outdoor pigeon coop into his basement. The patient often smelled the pigeons and noted feathers coming through the holes in the wall.

One of the key diagnostic features of hypersensitivity pneumonitis (HP) is the history of exposure to a potential offending antigen - in this case likely bird feathers-along with worsening upon reexposure to that antigen. HP is primarily a clinical diagnosis, and testing for serum precipitants has limited value, given the high false negative rate and the frequent lack of clinical symptoms accompanying positive testing. Bronchoalveolar lavage fluid may reveal lymphocytosis and reduced CD4:CD8 ratio. Crackles are commonly heard on examination, but in this case were likely not auscultated due to her obese habitus. The most important treatment is withdrawal of the offending antigen. Limited data suggest that corticosteroid therapy may be helpful in certain HP cases, including subacute, chronic and severe cases as well as patients with hypoxemia, significant imaging findings, and those with significant abnormalities on pulmonary function testing (PFT).

A hypersensitivity pneumonitis precipitins panel was sent with positive antibodies to $M$. faeni, T. Vulgaris, A. Fumigatus 1 and 6, A. Flavus, and pigeon serum. Her symptoms gradually improved within five days of oral prednisone $(60 \mathrm{mg})$. She was discharged home without dyspnea and normal oxygen saturation while breathing ambient air. A repeat echocardiogram after nighttime CPAP for one week demonstrated a right ventricular systolic pressure of $17 \mathrm{~mm} \mathrm{Hg}$ consistent with improved pulmonary hypertension.

Three weeks later, she returned to clinic for follow up. She had re-experienced dyspnea, cough, and wheezing, which improved when she was outdoors. She was afebrile, tachypneic, tachycardic, and her oxygen saturation was $92 \%$ on ambient air.

Her steroid-responsive interstitial lung disease and rapid improvement upon avoidance of the offending antigen is consistent with HP. The positive serum precipitins assay lends further credence to the diagnosis of HP, although serologic analysis with such antibody assays is limited by false positives and false negatives; further, individuals exposed to pigeons often have antibodies present without evidence of HP. History taking at this visit should ask specifically about further pigeon exposure: were the pigeons removed from the home completely, were heating-cooling filters changed, carpets cleaned, and bedding laundered? An in-home evaluation may be helpful before conducting further diagnostic testing.

She was admitted for oxygen therapy and a bronchoscopy, which showed mucosal friability and cobblestoning, suggesting inflammation. BAL revealed a normal CD4:CD8 ratio of 3; BAL cultures were sterile. Her shortness of breath significantly improved following a prolonged course of systemic steroids and removal from the triggering environment. PFTs improved with a FEV1/FVC ratio of 94 (105\% predicted), FVC of $2.00 \mathrm{~L}$ (66\% predicted), FEV1 of $1.88 \mathrm{~L}$ (69\% predicted) (Figure 3B). Her presenting symptoms of persistent cough and progressive dyspnea on exertion, characteristic $C T$, sterile BAL cultures, positive serum precipitants against pigeon serum, and resolution of her symptoms with withdrawal of the offending antigen were diagnostic of hypersensitivity pneumonitis due to pigeon exposure, also known as bird fancier's disease.

\section{COMMENTARY}

The patient's original presentation of dyspnea, tachypnea, and hypoxia is commonly associated with pediatric pneumonia and asthma exacerbations. ${ }^{1}$ However, an alternative diagnosis was suggested by the lack of wheezing, absence of fever, and recurrent presentations with progressive symptoms.

Hypersensitivity pneumonitis (HP) represents an exaggerated T-cell meditated immune response to inhalation of an offending antigen that results in a restrictive ventilatory defect and interstitial infiltrates. ${ }^{2}$ Bird pneumonitis (also known as bird fancier's disease) is a frequent cause of HP, accounting for approximately $65-70 \%$ of cases. ${ }^{3} \mathrm{HP}$, however, only manifests in a small number of subjects exposed to culprit antigens, suggesting an underlying genetic susceptibility. ${ }^{4}$ Prevalence estimates vary depending on bird species, county, climate, and other possible factors.

There are no standard criteria for the diagnosis of HP, though a combination of findings is suggestive. A recent prospective multicenter study created a scoring system for HP based on factors associated with the disease to aid in accurate diagnosis. The most relevant criteria included antigen exposure, recurrent symptoms noted within 4-8 hours after antigen exposure, weight loss, presence of specific IgG antibodies to avian antigens, and inspiratory crackles on exam. Using this rule, the probability that our patient has HP based on clinical characteristics was $93 \%$ with an area under the receiver operating curve of $0.93\left(96 \% \mathrm{Cl}\right.$ : 0.90-0.95). ${ }^{5}$ Chest imaging (high resolution $\mathrm{CT}$ ) often consists of a mosaic pattern of air trapping, as seen in this patient in combination with ground-glass opacities. ${ }^{6}$ Bronchoalveolar lavage (BAL) is sensitive in detecting lung inflammation in a patient with suspected HP. On BAL, a lymphocytic alveolitis can be seen, but absence of this finding does not exclude HP., 5 ,8 Pulmonary function tests (PFTs) may be normal in acute HP. When abnormal, PFTs may reveal a restrictive pattern and reduction in carbon monoxide diffusing capacity. ${ }^{7}$ However, BAL and PFT results are neither specific nor diagnostic of $\mathrm{HP}$; it is important 
to consider results in the context of the clinical picture.

The respiratory response to inhalation of the avian antigen has traditionally been classified as acute, subacute, or chronic. ${ }^{9}$ The acute response occurs within hours of exposure to the offending agent and usually resolves within 24 hours after antigen withdrawal. The subacute presentation involves cough and dyspnea over several days to weeks, and can progress to chronic and permanent lung damage if unrecognized and untreated. In chronic presentations, lung abnormalities may persist despite antigen avoidance and pharmacologic interventions. ${ }^{4,10}$ The patient's symptoms occurred over a six-month period which coincided with pigeon exposure and resolved during each hospitalization with steroid treatment and removal from the offending agent. Her presentation was consistent with a subacute time course of HP.

The dilated pulmonary artery, elevated right systolic ventricular pressure, and normal right ventricular function in our patient suggested pulmonary hypertension of chronic duration. Her risk factors for pulmonary hypertension included asthma, sleep apnea, possible obesity-hypoventilation syndrome, and HP-associated interstitial lung disease. ${ }^{11}$

The most important intervention in HP is avoidance of the causative antigen. Medical therapy without removal of antigen is inadequate. Systemic corticosteroids can help ameliorate acute symptoms though dosing and duration remains unclear. For chronic patients unresponsive to steroid therapy, lung transplantation can be considered. ${ }^{4}$

The key to diagnosis of HP in this patient-and to minimizing repeat testing upon the patient's recrudescence of symptoms-was the clinician's consideration that the major impetus for the patient's improvement in the hospital was removal from the offending antigen in her home environment. As in this case, taking time to delve deeply into a patient's environment-even by descending the basement stairs - may lead to the diagnosis.

\section{LEARNING POINTS}

- Consider hypersensitivity pneumonitis (HP) in patients with recurrent respiratory distress, offending exposure, and resolution of symptoms with removal of culprit antigen.
- The most important treatment of HP is removal of the offending antigen; systemic and/or inhaled corticosteroids are indicated until the full resolution of respiratory symptoms.

- Prognosis is dependent on early diagnosis and removal of offending exposures.

- Failure to treat HP might result in end-stage lung disease from pulmonary fibrosis secondary to long-term inflammation.

Disclosures: Dr. Manesh is supported by the Jeremiah A. Barondess Fellowship in the Clinical Transaction of the New York Academy of Medicine, in collaboration with the Accreditation Council for Graduate Medical Education (ACGME).

The authors declare no conflicts of interests.

\section{References}

1. Ebell MH. Clinical diagnosis of pneumonia in children. Am Fam Physician. 2010;82(2):192-193.

2. Cormier $Y$, Lacasse $Y$. Hypersensitivity pneumonitis and organic dust toxic syndrome. In: Malo J-L, Chan-Yeung M, Bernstein DI, eds. Asthma in the Workplace. Vol 32. Boca Raton, FL: Fourth Informa Healthcare; 2013:392-405.

3. Chan AL, Juarez MM, Leslie KO, Ismail HA, Albertson TE. Bird fancier's lung: a state-of-the-art review. Clin Rev Allergy Immunol. 2012;43(1-2):69-83. doi: 10.1007/s12016-011-8282-y.

4. Camarena A, Juárez $A$, Mejía $M$, et al. Major histocompatibility complex and tumor necrosis factor- $\alpha$ polymorphisms in pigeon breeder's disease. Am J Respir Crit Care Med. 2001;163(7):1528-1533. doi: 10.1164/ajrc$\mathrm{cm}$.163.7.2004023.

5. Lacasse $Y$, Selman M, Costabel U, et al. Clinical diagnosis of hypersensitivity pneumonitis. Am J Respir Crit Care Med. 2003;168(8):952-958. doi: 10.1164/ rccm.200301-137OC.

6. Glazer CS, Rose CS, Lynch DA. Clinical and radiologic manifestations of hypersensitivity pneumonitis. J Thorac Imaging. 2002;17(4):261-272.

7. Selman M, Pardo A, King TE Jr. Hypersensitivity pneumonitis: insights in diagnosis and pathobiology. Am J Respir Crit Care Med. 2012;186(4):314-324. doi: 10.1164/rccm.201203-0513Cl.

8. Calillad DM, Vergnon, JM, Madroszyk A, et al. Bronchoalveolar lavage in hypersensitivity pneumonitis: a series of 139 patients. Inflamm Allergy Drug Targets. 2012;11(1):15-19. doi: 10.2174/187152812798889330.

9. Richerson HB, Bernstein IL, Fink JN, et al. Guidelines for the clinical evaluation of hypersensitivity pneumonitis. Report of the Subcommittee on Hypersensitivity Pneumonitis. J Allergy Clin Immunol. 1989;84(5 Pt 2):839-844. doi: 10.1016/0091-6749(89)90349-7.

10. Zacharisen MC, Schlueter DP, Kurup VP, Fink JN. The long-term outcome in acute, subacute, and chronic forms of pigeon breeder's disease hypersensitivity pneumonitis. Ann Allergy Asthma Immunol. 2002;88(2):175-182. doi: 10.1016/S1081-1206(10)61993-X

11. Raymond TE, Khabbaza JE, Yadav R, Tonelli AR. Significance of main pulmonary artery dilation on imaging studies. Ann Am Thorac Soc. 2014;11(10):16231632. doi: 10.1513/AnnalsATS.201406-253PP. 